\title{
Microprocessor System for Temperature Investigation of AC Machines
}

\author{
Totyo Iliev \\ Department of Electric Power Supply and Equipment \\ Technical University of Gabrovo \\ Gabrovo, Bulgaria \\ totyo_iliev@abv.bg
}

\author{
Tsanko Karadzhov \\ Department of Mechanical and Precision Engineering \\ Technical University of Gabrovo \\ Gabrovo, Bulgaria \\ karadjov_st@abv.bg
}

\begin{abstract}
The paper is about four channel microprocessor system for temperature measurement during static and running mode. It incorporates microcontroller type PIC18F452 and employs sensors which convert temperature into voltage. In addition it features optional connection to a PC for storing and processing amassed data. Based on this system a new method is proposed for analysing overheating and cooling of individual parts of asynchronous electric machines. A set of differential equations is derived which later on are processed by MATHCAD software. Obtained results can be presented in tabular or graphic form. The outcome of resolving this task is in the relative ease with which it is possible to establish overheat of various machine parts. Similarly, such approach could be applied with other types of electric machines.
\end{abstract}

Keywords - Microprocessor, Microcontroller, Asynchronous Electric Machine, temperature sensor.

\section{INTRODUCTION}

Both circuits and equipment for temperature measurement find wide range of application in modern industry which in turn necessitates the design of such devices featuring an ever increasing number of function. Using sensor type LM35 a multichannel microprocessor system has been developed, capable of simultaneous measuring of four temperatures.

\section{METHOD FOR THERMAL INVESTIGATION OF ASYNCHRONOUS ELECTRIC MACHINES}

Two dissimilar approaches are possible to be used in determining the thermal condition of asynchronous machines. [1] [2] [3] [4]

According to the first, an equivalent thermal circuit is set up which usually includes four or five homogeneous bodies such as:

$$
\begin{aligned}
& 1 \text { - stator pack; } \\
& 2 \text { - rotor; } \\
& 3 \text { - stator coil; }
\end{aligned}
$$

All these elements with the exception of the last one do generate thermal losses whose values become evident from experimental measurements or calculations. Each body (element) has its own thermal capacity and all of them are interconnected by thermal conductivity which is conductive and convective.

Convective conductivities are largely dependent on the speed of rotation and, consequently, their values during performance are far more different than the values taken during pause.

With operation mode S4 which is usual for asynchronous machines start-off losses are also to be taken into account. This task entails the set-up of a system of four differential equations and is further to be solved by means of MATHCAD. This results in obtaining thermal dependence for each element from the moment of the transition period start and the quasi-initiation mode.

Such approach is appropriate for practical investigations of thermal processes within the machine. With real functioning machine it is possible to conduct thermal investigations and define thermal parameters with greater accuracy. That, in general, is the essence of this approach.

The above equivalent thermal circuit which contains four homogeneous elements is presented in Fig. 1 


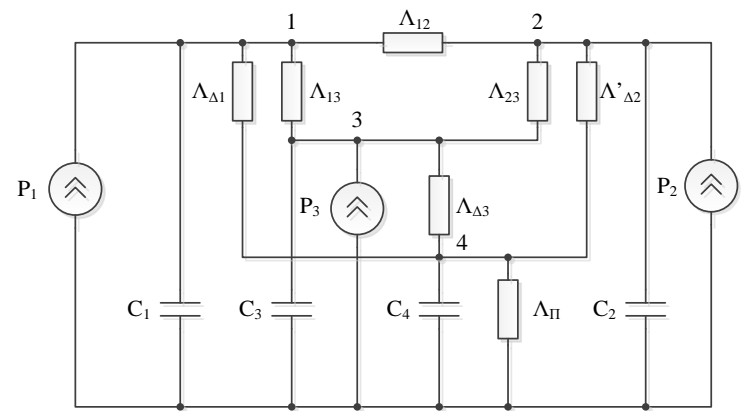

Fig. 1. Equivalent thermal circuit

P1, P2 and P3 are the mean values of losses during operation time $\mathrm{t}$ for each operation mode.

$\boldsymbol{\Lambda}_{\mathbf{1 2}}$ - thermal conductivity of the air gap;

$\boldsymbol{\Lambda}_{\mathbf{1 3}}$ - thermal conductivity between copper and steel via channel insulation;

$\boldsymbol{\Lambda}_{\boldsymbol{\Delta} \mathbf{1}}-$ thermal conductivity of contact gap between the pack and the frame;

$\boldsymbol{\Lambda}_{\boldsymbol{\Delta \mathbf { 2 }}}^{\prime}$ - thermal conductivity of rotor to the frame via the shaft;

$\boldsymbol{\Lambda}_{\mathbf{2 3}}$ - equivalent thermal conductivity between the second and third body(element);

$\boldsymbol{\Lambda}_{\mathbf{\Delta} 3}$ - equivalent thermal conductivity between the third body and the frame;

$\boldsymbol{\Lambda}_{\Pi}$ - thermal conductivity of the frame to the ambience;

$\mathbf{C}_{1}, \mathbf{C}_{2}, \mathbf{C}_{3}$ и $\mathbf{C}_{\mathbf{4}}-$ thermal capacities of all four bodies;

$\boldsymbol{\theta}_{1}, \boldsymbol{\theta}_{2}, \boldsymbol{\theta}_{3}$, и $\boldsymbol{\theta}_{\mathbf{4}}$ - overheating of all four bodies.

These parameters are determined by way of experiment and are derived from the measurements made with the developed microprocessor system.

The proposed equivalent circuit allows to compile the following system of differential equations:

$$
\begin{aligned}
& \frac{d \theta_{1}}{d t}=-\frac{\Lambda_{11}}{C_{1}} \theta_{1}+\frac{\Lambda_{12}}{C_{2}} \theta_{2}+\frac{\Lambda_{13}}{C_{1}} \theta_{3}+ \\
& +\frac{\Lambda_{\Delta 1}}{C_{1}} \theta_{4}+\frac{1}{C_{1}} P_{1}\left(1+\alpha_{1} \theta_{1}\right) \\
& \frac{d \theta_{2}}{d t}=\frac{\Lambda_{12}}{C_{2}} \theta_{1}-\frac{\Lambda_{22}}{C_{2}} \theta_{2}+\frac{\Lambda_{23}}{C_{2}} \theta_{3}+ \\
& +\frac{\Lambda_{\Delta 2}}{C_{2}} \theta_{4}+\frac{1}{C_{2}} P_{2}\left(1+\alpha_{2} \theta_{2}\right) \\
& \frac{d \theta_{3}}{d t}=\frac{\Lambda_{13}}{C_{3}} \theta_{1}+\frac{\Lambda_{23}}{C_{3}} \theta_{2}-\frac{\Lambda_{33}}{C_{3}} \theta_{3}+ \\
& +\frac{\Lambda_{\Delta 3}}{C_{3}} \theta_{4}+\frac{1}{C_{3}} P_{3}\left(1+\alpha \theta_{3}\right) \\
& \frac{d \theta_{4}}{d t}=\frac{\Lambda_{\Delta 1}}{C_{4}} \theta_{1}+\frac{\Lambda_{\Delta 2}^{\prime}}{C_{4}} \theta_{2}+\frac{\Lambda_{\Delta 3}}{C_{4}} \theta_{3}- \\
& -\frac{\Lambda_{44}}{C_{4}} \theta_{4}-\frac{\Lambda_{44}}{C_{4}} \theta_{4} \\
& \Lambda_{11}=\Lambda_{12}+\Lambda_{13}+\Lambda_{\Delta 1}
\end{aligned}
$$

$$
\begin{aligned}
& \Lambda_{22}=\Lambda_{12}+\Lambda_{23}+\Lambda_{\Delta 2}^{\prime} \\
& \Lambda_{22}+\Lambda_{13}+\Lambda_{23}+\Lambda_{03} \\
& \Lambda_{44}+\Lambda_{\Delta 1}+\Lambda_{02}^{\prime}+\Lambda_{\Delta 3}+\Lambda_{\Pi}
\end{aligned}
$$

It should be solved with zero initial conditions. By using Mathcad software we get the solutions for this set of equations: $\theta_{1}, \theta_{2}, \theta_{3}$ and $\theta_{4}$. The obtained overheating values $\theta_{1}, \theta_{2}, \theta_{3}$ and $\theta_{4}$ for $\mathrm{t}=\mathrm{t}_{1}$ are initial conditions for solving the set of equations. Solutions obtained for overheating $\theta_{1}, \theta_{2}$, $\theta_{3}$ and $\theta_{4}$ for $\mathrm{t}=\mathrm{t}_{2}$ are in turn the initial conditions for solving the set of equations until quasi-established mode is reached. It is evident that overheating values $\theta_{1}, \theta_{2}, \theta_{3}$ and $\theta_{4}$ even in quasi-established mode (S3) pulse in so narrow interval that they could be regarded as constants. This is all the more valid for (S4) mode. In this way the notion of "temperature source" is reached. Power sources can be substituted with overheating sources. Moreover, for this particular case it is convenient to apply the method of average parameters which is stated in [5] [6] [7]

\section{PRINCIPLE ELECTRIC DIAGRAM OF MICROPROCESSOR SYSTEM}

The diagram is presented on Fig.2

LM35 stand for precision temperature sensors wherein output voltage depends linearly on temperature [8]. They feature a measuring range of:
LM35, LM35A
from $-55^{\circ} \mathrm{C}$ to $+150^{\circ} \mathrm{C}$
LM35A, LM35CA
from $-40^{\circ} \mathrm{C}$ to $+110^{\circ} \mathrm{C}$
LM35D
from $0^{\circ} \mathrm{C}$ to $+100^{\circ} \mathrm{C}$

Output voltage is changed by $10 \mathrm{mV} /{ }^{\circ} \mathrm{C}$.

$$
U_{t}=0,01 t,[V]
$$

Voltage is transferred from sensors' outputs to operation amplifiers LM358 with amplification factor:

$$
A=1+\frac{R 2}{R 1}=5
$$

In this way the entire measuring range of controller's analogue-digital converter is utilized.

Voltage supplied to analogue-digital converter is

$$
U_{O U}=5.0,01 t,[V]
$$

To effectively enable device operation a microcontroller PIC18F452 is used, which features 10-bit analogue-todigital converter [9]. The controller has enough number of input/output ports and sufficient storage to locate the control program in.

Threshold voltages which determine the converter's measuring range are $\mathrm{V}_{\mathrm{REF}}=0 \mathrm{~V}$ and $\mathrm{V}_{\mathrm{REF}+}=5,12 \mathrm{~V}$. Input analogue signal which is supplied to the converter is presented by $2^{10}$ or 1024 levels. 
Environment. Technology. Resources. Rezekne, Latvia Proceedings of the $13^{\text {th }}$ International Scientific and Practical Conference. Volume 3, 110-115

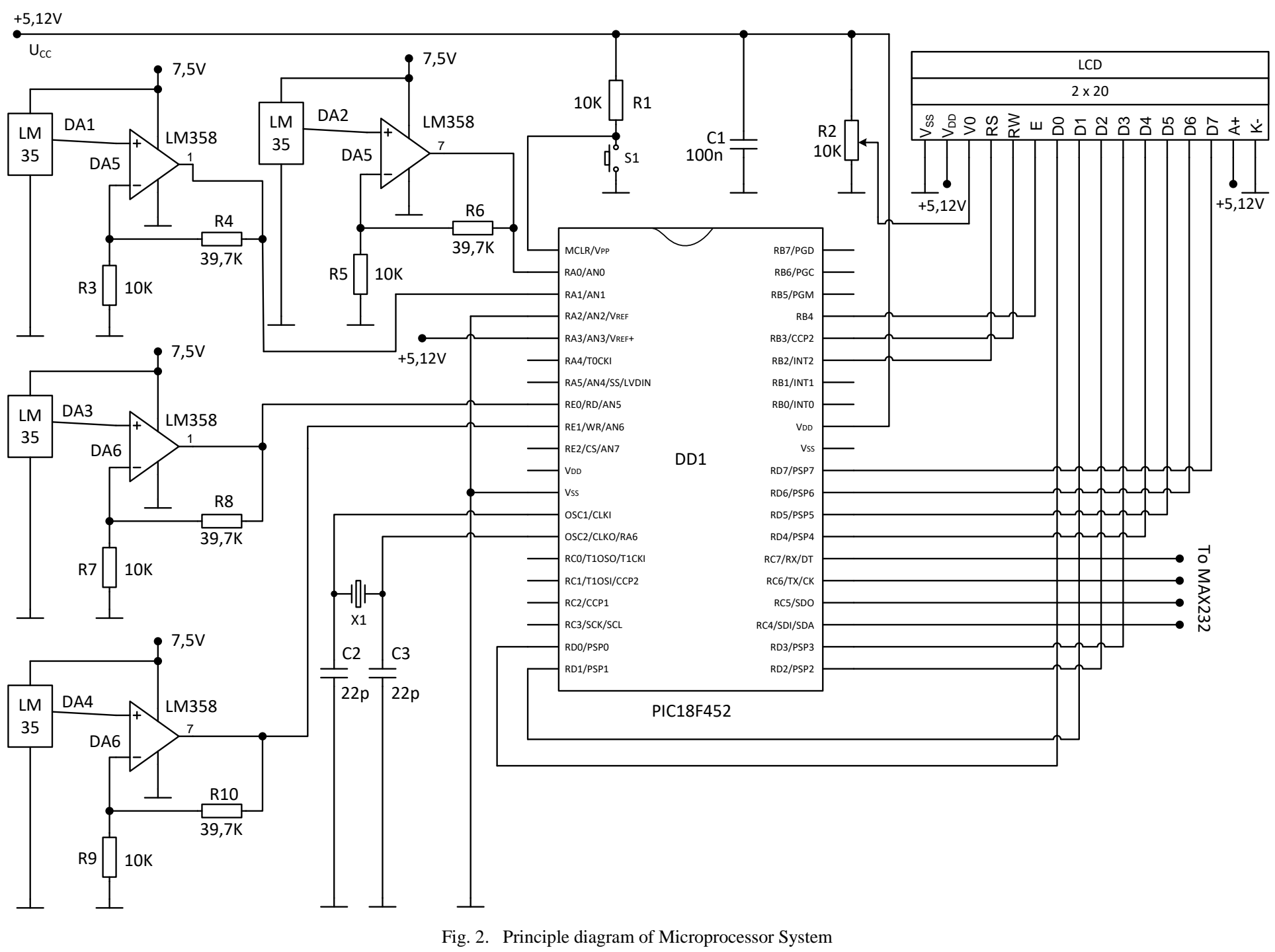


The step of voltage increment is defined by the formula:

$$
\Delta U=\frac{U_{C C}}{2^{10}}=\frac{5,12}{1024}=5 \mathrm{mV}
$$

Supply voltage is selected to be $5.12 \mathrm{~V}\left(\mathrm{U}_{\mathrm{CC}}\right)$ for the step to be $5 \mathrm{mV}$.

The function diagram of microprocessor system is composed of four measuring channels each of which incorporates temperature sensor LM35 and measuring amplifier LM358. Voltage is supplied from amplifiers' outputs to the four leads of controller: RA0/AN0, RA1/AN1, RE0/AN5, RE1/AN6 which are configured as analogue inputs. Inputs are switched over. At any point in time voltage is measured at only one input.

Fig. 3 shows a picture of the measuring system.

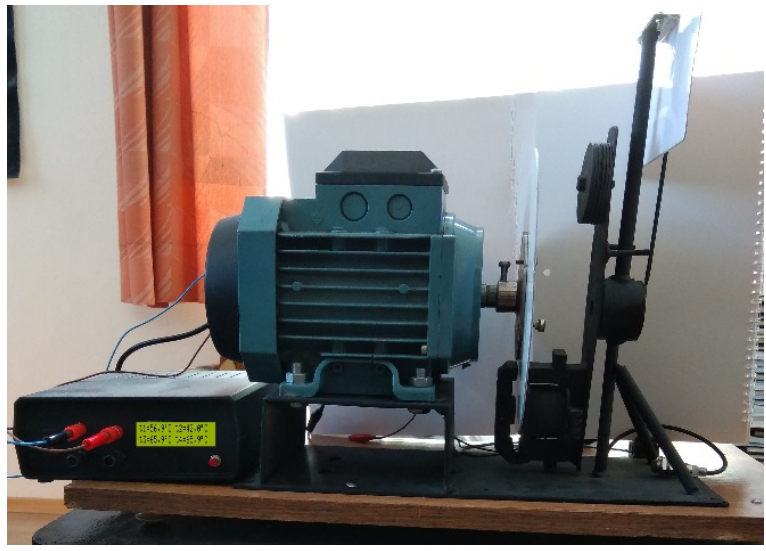

Fig. 3. Measuring system

Fig. 4 shows how the measuring microprocessor system is linked to a personal computer.

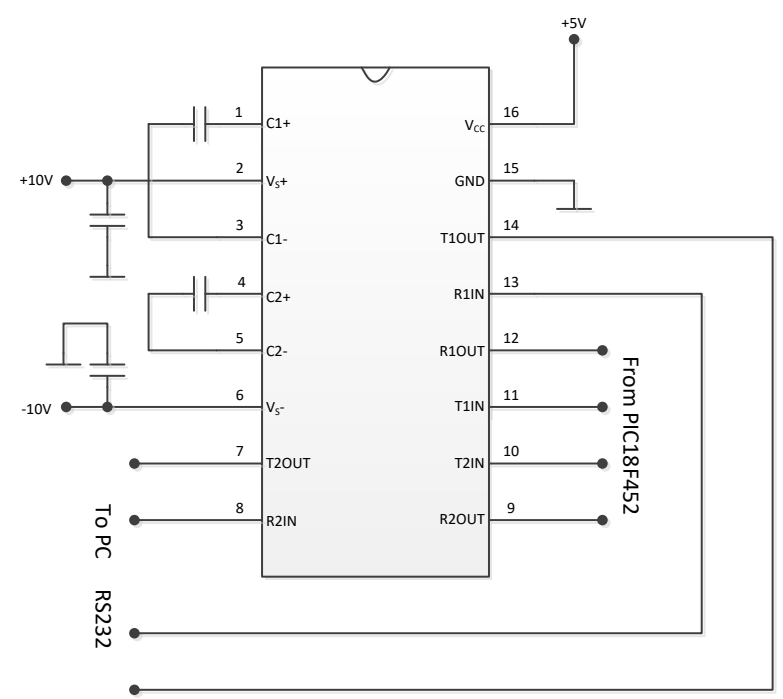

Fig. 4. Microcontroller linked to a PC

Flow chart (block schematic) of program's algorithm

This flow chart is presented in Fig.7 and Fig 8. At the beginning the controller is initialized, constants and variables are defined and storage locations are allocated for them in the data storage. The next step of the program concerns determination of input/output ports. Controller ports are given assignments as digital inputs, analogue inputs and digital outputs. PIC18F452 features four internal module timers. Only Timer 0 is used by means of which program time-lags are assigned and used to synchronize operation of microcontroller and RS232 of computer. The main program is a continuous cycle which is interrupted only when voltage measurement, done by analogue-to-digital converter, is completed.

Processing of each measurement is executed by a subroutine which is started whenever an interruption by analogue-digital converter occurs which signals that measurement has completed.

Input is made followed by processing of measurement result which includes hardware multiplication of measurement value by the assigned constant and conversion of the result from hexadecimal into binary-decimal code. The second stage of the subroutine involves measurement data transfer to the PC. Finally, next measurement is started in the subroutine followed by a return to the main program.

Fig 5 visualizes the dependence of voltages at the output ports of operation amplifiers.

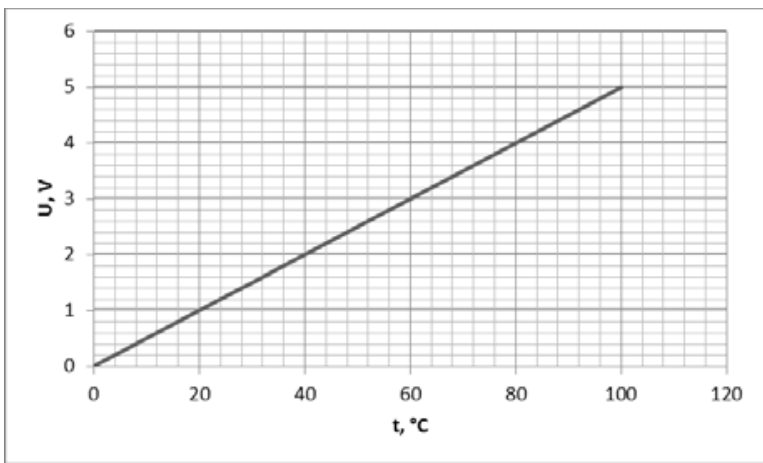

Fig. 5. Transmission characteristic of temperature sensor

\section{OPERATIONAL FEATURES OF THE ELECTRONIC DEVICE FOR TEMPERATURE MEASUREMENT}

1. Measurement of four different temperatures on various parts of any machine;

2. Measurement data transfer to a PC;

3. Possible control of four external electric circuits depending on the assigned threshold temperature values;

\section{SUBROUTINE FOR PROCESSING ANALOGUE-DIGITAL CONVERTER INTERRUPTION}

At the initiation of subroutine a selection of analogue input is made followed by processing of measurement result which includes hardware 
Environment. Technology. Resources. Rezekne, Latvia Proceedings of the $13^{\text {th }}$ International Scientific and Practical Conference. Volume 3, 110-115

multiplication of measurement value by the assigned constant and conversion of the result from hexadecimal into binary-decimal code. The second stage of the subroutine involves measurement data transfer to the PC. Finally, next measurement is started in the subroutine followed by a return to the main program.

The experimental results are given in fig. 7

Fig 6 visualizes the dependence of temperature $(\theta)$ at the time of operation $(\mathrm{t})$.

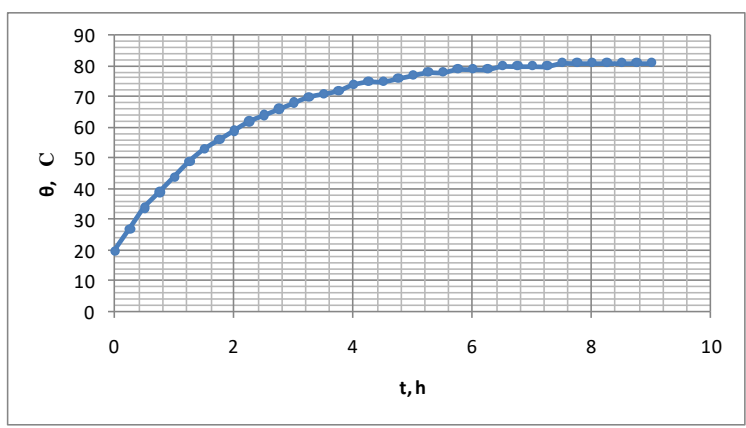

Fig. 6. Experimental results

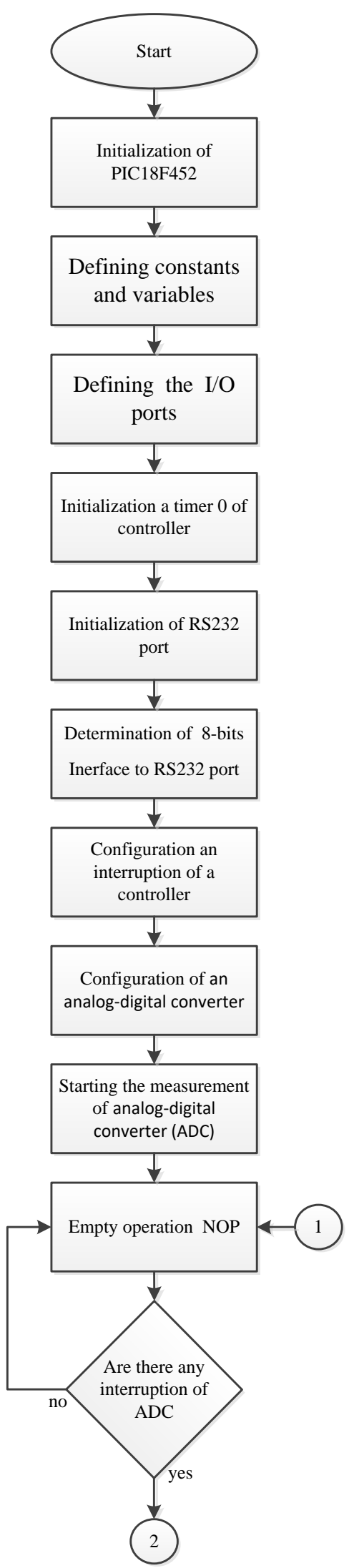

Fig. 7. Algorithm of main program 


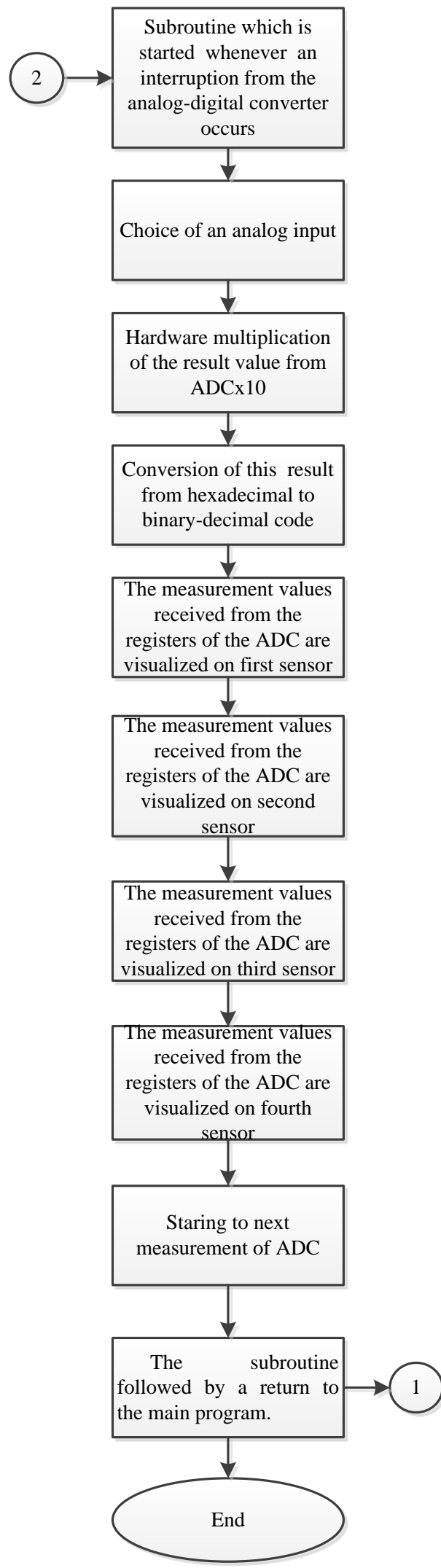

Fig. 8. Algorithm of main program

\section{CONCLUSION}

Generally, the microprocessor system is applicable to electric machines which vary in power capacity and purpose of use allowing to be linked to a PC. In this way it is possible to amass a data array later to be used for thermal analysis. The system is mobile and attachable to every electric machine; its computer link allows for measurement processing with various software, visualization of results and signalling in case of exceeding temperature values.

\section{REFERENCES}

[1] A. Dumcius, V. Augutis and D. Gailius, "The Approximation Aspects of Characteristics of Semiconductor Temperature Sensors," Electronics and Electrical Engineering, no. 6(112), pp. 47-50, 2011.

[2] O. Krejcar, I. Spicka and R. Frischer, "Implementation of FullFeatured PID Regulator in Microcontrollers," Electronics and Electrical Engineering, no. 7(113), pp. 77-82, 2011.

[3] R. Hajovsky and M. Pies, "Complex Measuring System for Longtime Monitoring and Visualization of Temperature and Toxic Gases Concentration," Electronics and Electrical Engineering, no. 6(122), pp. 129-132, 2021.

[4] V. Markevicius and D. Navikas, "Adaptive ThermoCompensation of Magneto-Resistive Sensor," Electronics and Electrical Engineering, no. 8(114), pp. 43-46, 2011.

[5] J. Leskauskaite and A. Dumcius, "The Selection of Thermistors for the Temperature Measurement Gear," Electronics and Electrical Engineering, no. 5(111), pp. 59-62, 2011.

[6] B. Gilev, N. Hinov and H. Ibrishimov, "Mathematical Model of Induction Heating with Heat Transfer of Cylindrical Body for Pressing Treatment," in 2019 International Conference on High Technology for Sustainable Development, Sofia, 2019, DOI: 10.1109/HiTech48507.2019.9128250

[7] N. D. Mazharov, S. M. Hristov, D. A. Dichev and I. S Zhelezarov, "Some problems of dynamic contactless charging of electric vehicles," Acta Polytechnica Hungarica, vol. 14, no. 4, pp. 7-26, 2017, DOI: 10.12700/APH.14.4.2017.4.1

[8] [Online]. Available: http://www.ti.com/lit/ds/symlink/lm35.pdf.

[9] [Online]. Available: http://ww1.microchip.com/downloads/en/devicedoc/39564c.pdf. 